\title{
Elevation Resolved Elemental Mapping With SEM/EDS
}

\author{
John Konopka*, Mark Betts** \\ *Thermo Fisher Scientific, 5225 Verona Road, Madison, WI 53711 \\ **Hitachi HTA, 5100 Franklin Drive, Pleasanton, CA 94588
}

In this work we combine sample elevation masks and elemental composition masks to automatically find just those areas characterized by both a given composition and elevation. Applications include differentiating contaminants from substrates when they share common elements and evaluating manufactured materials where sample height is important as in abrasives. Both SEM and EDS data are required as EDS alone does not measure elevation and SEM alone does not measure composition. The goal is a technique for routine evaluation of materials with rough surfaces.

It is possible to measure sample elevation using four images gained from the four independent segments of a four quadrant backscatter electron(BSE) detector[1] in an SEM. Figure 1 shows a BSE image of a wire attached to a bond pad of an integrated circuit. Figure 2 shows an elevation profile taken along the blue line shown in Figure 1. Figure 3 shows an image in which the grey scale encodes sample height. Brighter areas are higher above the substrate.

Spectral imaging collects an x-ray map by storing a full x-ray spectrum at each point in an array which spans a portion of a sample. From this Multivariate Spectral Analysis[2] finds self consistent patterns in the data. A clustering algorithm converts this result to binary masks defining areas of the sample that have similar composition. Together these three technologies allow an analyst to automatically find areas of similar composition. The use of a silicon drift detector(SDD) allows this process to proceed very rapidly[3]. Figure 4 is the automatically determined mask showing the distribution of $\mathrm{Al}$ in this sample. Note that it does not differentiate the wire from the pad.

Figure 5 is obtained by thresholding the image in Figure 3 isolating just the parts of the sample at the level of the flat surface of the wire.

The final result in Figure 6 is obtained by performing a logical AND of each corresponding pair of points in figures 4 and 5. This trivial example was chosen to exhibit the technique clearly.

The next sample consists of a mixture of $\mathrm{NaCl}$ and $\mathrm{KCl}$ resting on a bed of $\mathrm{NaCl}$ and carbon. Figures 7, 8 and 9 show the BSE image, height profile and the height encoded image respectively.

Figures 10, 11 and 12 show the $\mathrm{Na}, \mathrm{Cl}$ and $\mathrm{K}$ x-ray distribution maps respectively.

Figure 13 shows the $\mathrm{NaCl}$ mask determined by MVS and clustering. Figure 14 is the threshold mask generated from figure 9. Figure 15 is the result which finds just the area of the sample consisting of $\mathrm{NaCl}$ and which is above the level of the substrate. The use of dual opposed detectors would improve on this result by reducing shadowing artifacts producing unambiguous data for all points.

A Hitachi S-3700 SEM was used to obtain the BSE images and 3D images. A Thermo Scientific NS7 EDS system with a single Ultradry SDD was used to collect the x-ray maps and composition masks. Applescript and GraphicConverter were used to prototype the thresholding and logic operations.

References

[1] I. C. Carlsen, Scanning 7 (1985) 169-177

[2] P.G. Kotula, M.R. Keenan, J.R. Michael, Microsc. Microanal. 9 (2003) 1-19

[3] JJ McCarthy et al., Microsc. Microanal. 14 (Suppl. 2) (2008) 114 


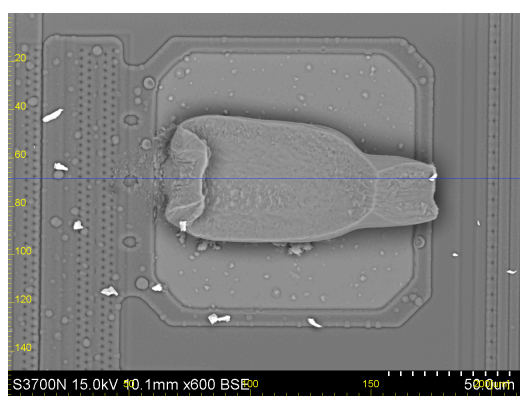

Figure 1. BSE image

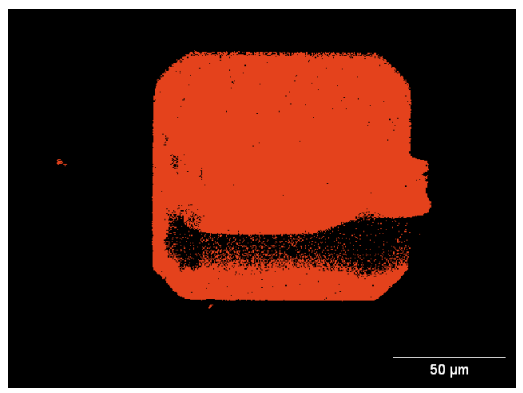

Figure 4. Al distribution mask

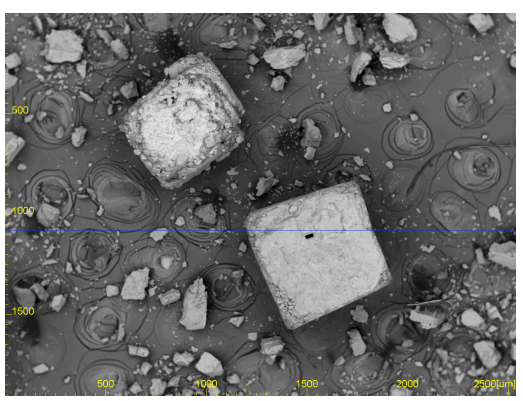

Figure 7. BSE image of salts

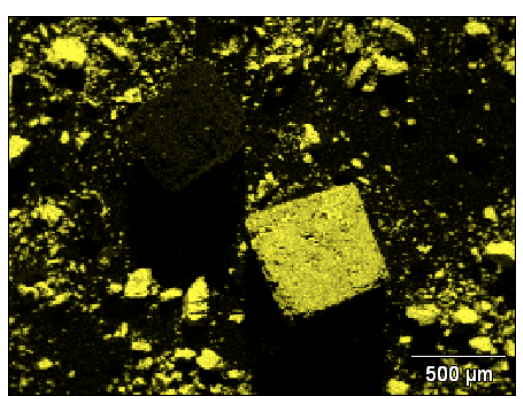

Figure 10. Na distribution map

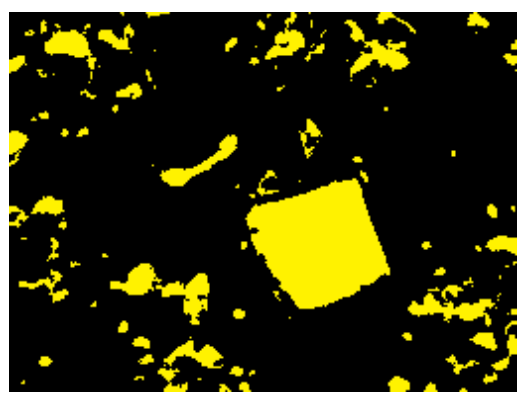

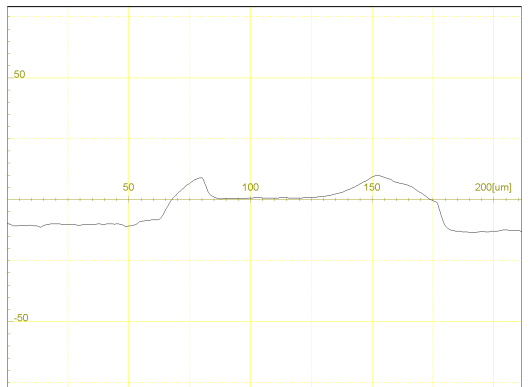

Figure 2. Elevation profile

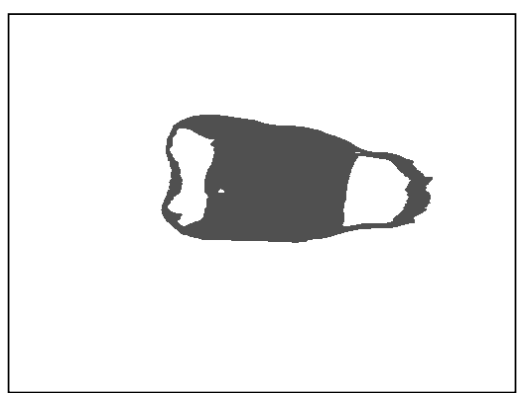

Figure 5. Elevation threshold mask

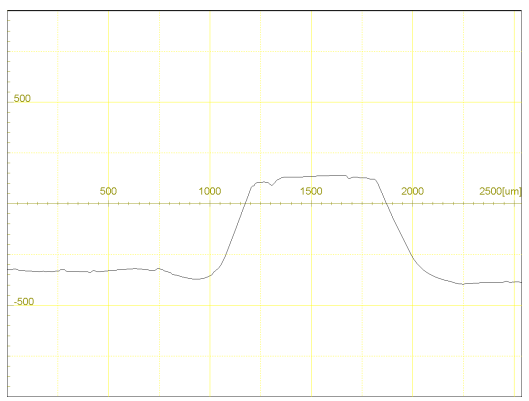

Figure 8. Elevation profile

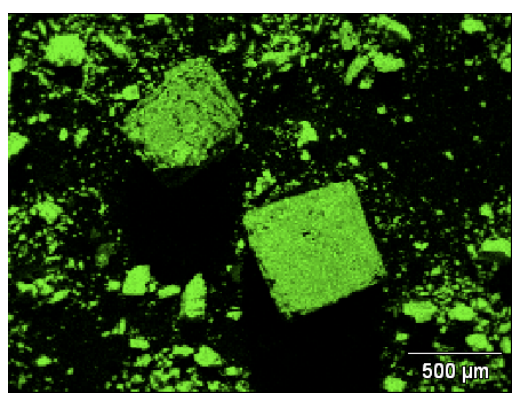

Figure 11. $\mathrm{Cl}$ distribution map

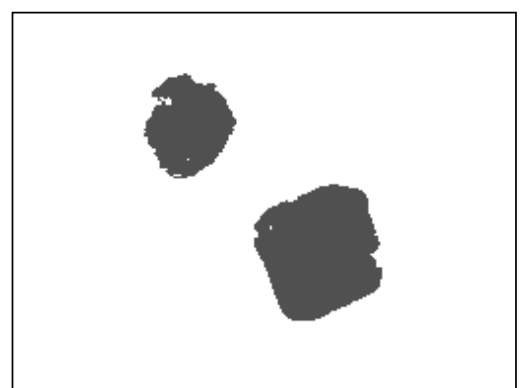

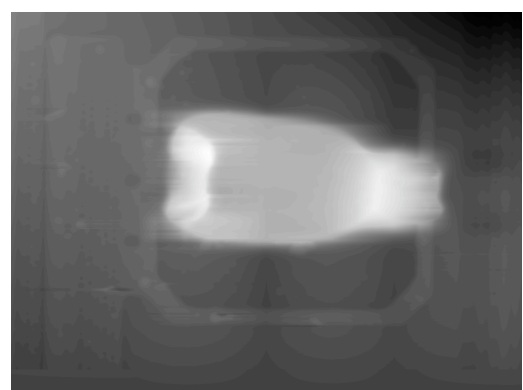

Figure 3. Height encoded image

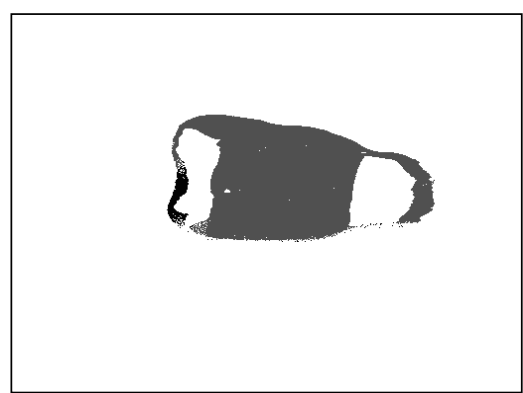

Figure 6. Elevated Al

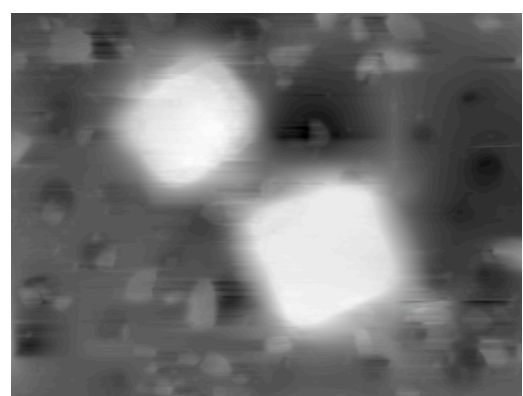

Figure 9. Height encoded image

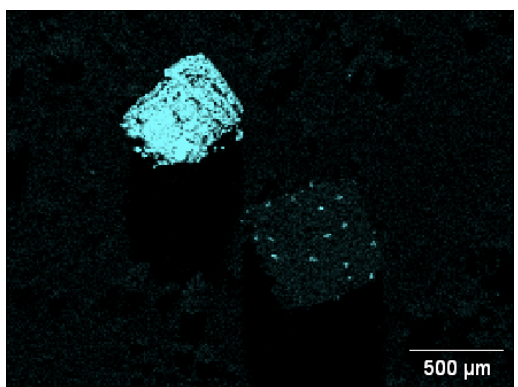

Figure $12 . \mathrm{K}$ distribution map

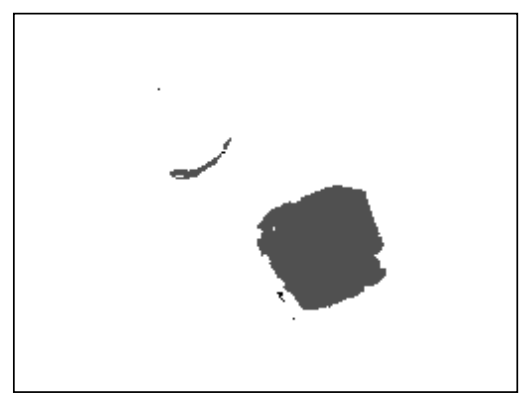

Figure 13. $\mathrm{NaCl}$ distribution mask Figure 14. Elevation threshold mask Figure 15. Elevated $\mathrm{NaCl}$ https://doi.org/10.1017/51431927609096895 Published online by Cambridge University Press 